Ágora Rev. Cient. 2017; 04(02):e3

\title{
Percepción de los padres y calidad en el cuidado de enfermería a pacientes pediátricos en un hospital en Perú, 2017
}

\author{
Parent's perception and nursing care quality in pediatric patients into a Hospital in Peru, 2017 \\ Lizeth Margarita Camargo Gonzales ${ }^{1, a}$, Violeta Taipe Huamán ${ }^{1, a}$, Victor Humberto Chero Pacheco 1,b
}

RESUMEN

Objetivo: Determinar la percepción de los padres sobre la calidad del cuidado de enfermería a pacientes pediátricos en un hospital en Perú, 2017. Materiales y Métodos: El estudio fue cuantitativo, aplicado y de nivel descriptivo con diseño no experimental-transversal. La muestra estuvo conformada por 110 padres de familia a quienes se aplicó previamente el consentimiento informado. El muestreo fue no probabilístico. Los datos fueron procesados y representados por medio de tablas de frecuencia. Resultados: Considerando las dimensiones del estudio, del total de padres, el 88,2\% tiene una percepción favorable acerca de la dimensión técnico-científica y el 11,8\% tiene una percepción desfavorable. Respecto a la dimensión humana, el 94,5\% de padres tiene una percepción favorable y el $5,5 \%$, tiene una percepción desfavorable. De acuerdo a la dimensión entorno, se evidencia que, el $93,6 \%$ de padres tiene una percepción favorable y el 6,4\%, tiene una percepción desfavorable. El $90 \%$ de los padres tiene una percepción favorable acerca de la calidad en el cuidado, mientras que el 10\% de los padres tiene una percepción desfavorable. Conclusión: La percepción de los padres de familia sobre la calidad en el cuidado de enfermería es favorable en la mayoría de casos.

Palabras clave: percepción de los padres, calidad en el cuidado de enfermería.

\begin{abstract}
Objective: To determine parents' perception regarding Nursing care quality in pediatric patients into a hospital in Peru, 2017. Materials and Methods: The study was applied, quantitative, and descriptive. Also, it was considered a cross-sectional and non-experimental design. The sample included 110 participants who were asked for permission by using an informed consent. Participants were selected by the use of The Nonprobability Sampling. Data analysis was developed using frequency charts. Results: According to research dimensions, parents' perception is favorable in $88,2 \%$ of cases and it is unfavorable in $11,8 \%$ of cases according to the technical and scientific dimension. It is evident that parents' perception is favorable in $94,5 \%$ of cases and it is unfavorable in $5,5 \%$ of cases according to the human dimension. It is evident that parents' perception is favorable in $93,6 \%$ of cases and it is unfavorable in $6,4 \%$ of cases according to the environment dimension. It is evident that parents' perception is favorable in $90 \%$ of cases and it is unfavorable in $10 \%$ of cases according to the nursing care. Conclusion: Parents' perception about nursing care quality is favorable in most of the cases.
\end{abstract}

Key words: parents' perception, Nursing care quality.

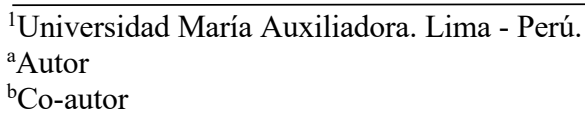

\section{INTRODUCCIÓN}

El personal de enfermería participa en actividades de identificación de problemas, apoyo en el establecimiento de un diagnóstico, labores de intervención, evaluación de los impactos en los individuos; es decir, sus actividades priorizan el cuidado de los pacientes. Constantemente se llevan a cabo procesos de autoevaluación, con los cuales pueden identificarse debilidades que son susceptibles a corrección, ya que se asume la responsabilidad frente a los individuos vulnerables en las instituciones de salud.

Los individuos comprometidos por situaciones complejas, requieren ser derivados a establecimientos de salud. A partir de ese instante, 
es necesario tomar en cuenta ciertas características relacionadas. Como ejemplos pueden considerarse, lo concerniente al motivo por el cual se acude al servicio, la conducta de los sujetos, el nivel cultural de los mismos, la edad de los pacientes. Además, es importante destacar el entorno dichos pacientes por representar un papel fundamental durante su tratamiento $\mathrm{y}$ recuperación. Es indispensable comprender las características del entorno familiar o social de los sujetos. Si esto no es tomado en cuenta, los resultados no serán favorables.

Los procedimientos orientados a la búsqueda del bienestar del paciente y a los miembros de su entorno, serán desarrollados según parámetros de calidad tal como es señalado por la Organización Mundial de la Salud (OMS), en donde se destaca el ejercicio de la Enfermería como valioso recurso para la salud, considerando que los cuidados deben ofrecer un servicio competente, diferente al de otros grupos profesionales y de utilidad para las personas que lo requieren (1).

Se debe considerar el aspecto emocional o psicológico además del físico. Se sabe que la percepción de los sujetos respecto a la atención, predispone hacia las conductas adecuadas y el establecimiento de procesos comunicativos eficientes, con lo cual es posible el intercambio de información con la finalidad de conseguir el bienestar de los pacientes y su entorno social.

La percepción así considerada no solo por los pacientes sino también por los miembros de su entorno, facilitarán la aplicación de estrategias apropiadas, favoreciendo la aceptación y logrando la satisfacción esperada como parte de los objetivos durante el cuidado y en el tiempo posterior a éste.

Además, a pesar de entender a la percepción como una actividad aparentemente sencilla y desarrollada incluso de forma espontánea y natural, constituye un problema en sí mismo y requiere una explicación que involucre los elementos relacionados a esta. Las explicaciones respecto a las características de la percepción pueden considerarse desde un enfoque multidisciplinario a partir de las ciencias de la naturaleza por ejemplo, ya que se consideran los elementos del entorno o, también desde la perspectiva de la neurofisiología la cual señala cómo funcionan los órganos sensoriales por los que captamos el mundo. Los sujetos no suelen percatarse de los problemas de la percepción a no ser que le falten los estímulos o padezca algún tipo de déficit sensorial (2).

Por lo señalado, la percepción puede ser estudiada desde diversos puntos de vista $\mathrm{y}$, probablemente, la consideración de todos ellos sea importante a la hora de dar con una explicación precisa de la misma. Pero, es preciso delimitar, lo mejor posible, el tipo de problemas que interesan en cada caso (3).

Respecto a la calidad en los servicios de salud, se señala que es la obtención del máximo beneficio del usuario mediante la aplicación del conocimiento y tecnología más avanzada teniendo en cuenta los requerimientos del paciente, así como las capacidades y limitaciones de recursos de la institución de acuerdo a los valores sociales imperantes (4).

La calidad, considera aspectos o dimensiones referidas a lo siguiente: En primer lugar, lo técnico y científico, que hace referencia a la capacidad de los proveedores de utilizar el más avanzado nivel de conocimiento existente para abordar los problemas de salud. Es la dimensión que mejor se entiende y más frecuentemente se mide, significa atender de forma científica las necesidades sanitarias. Luego, se considera lo humano, que en la profesión de enfermería es prioritario, ya que se da cuidado al paciente. Del profesional de enfermería depende la integridad del paciente, además del respeto que se le brinde, la cordialidad y haciendo práctica del código de ética en la atención al paciente, ya que es un ser holístico, íntegro y espiritual. Finalmente, se toma en cuenta el entorno, referido a las facilidades que la institución dispone para la mejor prestación de los servicios (5).

\section{MATERIALES Y MÉTODOS}

El estudio realizado considera el carácter cuantitativo, aplicado y descriptivo, además de ser un estudio no experimental, transversal. La muestra de estudio consideró 110 padres de familia cuyos hijos se hallaban hospitalizados en el Hospital San Juan de Lurigancho durante el mes de abril del año 2017. La información respecto a la variable: Percepción de los padres respecto al cuidado de enfermería de pacientes pediátricos, fue registrada por medio de un cuestionario elaborado en base a los indicadores de las dimensiones: técnica científica, humana y entorno. Se desarrolló el procesamiento de datos por medio del programa estadístico SPSS considerando el análisis estadístico descriptivo.

\section{RESULTADOS}

Respecto a la percepción de los padres sobre la calidad de cuidado de enfermería en la dimensión técnico-científica, el $88,2 \%$ de los encuestados respondieron "Siempre". El 11,8\% de encuestados respondieron "A veces". (Tabla 1). 
Tabla 1. Percepción de los padres respecto a la calidad de cuidado de enfermería en la dimensión técnico-científica.

\begin{tabular}{cccc}
\hline & Nivel & $\mathbf{N}^{\circ}$ & Porcentaje \\
\hline A veces & 13 & 11,8 \\
\multirow{2}{*}{ Siempre } & 97 & 88,2 \\
\hline \multicolumn{2}{r}{ Total } & $\mathbf{1 1 0}$ & $\mathbf{1 0 0 , 0}$ \\
\hline
\end{tabular}

Respecto a la percepción de los padres sobre la calidad de cuidado de enfermería en la dimensión humana, se observa que el $94,5 \%$ de los encuestados respondieron "Siempre". E1 5,5\% de encuestados respondieron "A veces". (Tabla 2).

Tabla 2. Percepción de los padres respecto a la calidad de cuidado de enfermería en la dimensión humana.

\begin{tabular}{lccc}
\hline & Nivel & $\mathbf{N}^{\circ}$ & Porcentaje \\
\hline A veces & 6 & 5,5 \\
Siempre & 104 & 94,5 \\
\hline \multicolumn{2}{r}{ Total } & $\mathbf{1 1 0}$ & $\mathbf{1 0 0 , 0}$ \\
\hline
\end{tabular}

Respecto a la percepción de los padres sobre la calidad de cuidado de enfermería en la dimensión entorno, se observa que el $93,6 \%$ de los encuestados respondieron "Siempre". El 6,4\% de encuestados respondieron "A veces". (Tabla 3).

Tabla 3. Percepción de los padres respecto a la calidad de cuidado de enfermería en la dimensión entorno.

\begin{tabular}{cccc}
\hline & Nivel & $\mathbf{N}^{\circ}$ & Porcentaje \\
\hline A veces & 7 & 6,4 \\
\multirow{2}{*}{ Siempre } & 103 & 93,6 \\
\hline & Total & $\mathbf{1 1 0}$ & $\mathbf{1 0 0 , 0}$ \\
\hline
\end{tabular}

Respecto a la percepción de los padres sobre la calidad durante el cuidado de enfermería a pacientes pediátricos en el Hospital San Juan de Lurigancho, 2017. Se observa que el $90 \%$ de los encuestados respondieron "Siempre". El 10\% de encuestados respondieron "A veces". (Tabla 4).

Tabla 4. Percepción de los padres respecto a la calidad de cuidado enfermería en la dimensión entorno.

\begin{tabular}{|c|c|c|}
\hline Nivel & $\mathbf{N}^{\circ}$ & Porcentaje \\
\hline A veces & 11 & 10,0 \\
\hline Siempre & 99 & 90,0 \\
\hline Total & 110 & 100,0 \\
\hline
\end{tabular}

\section{DISCUSIÓN}

Según la dimensión técnico-científica, la mayoría de padres percibe una buena atención. Esto se representa por el $88,2 \%$ de individuos quienes eligieron la opción "siempre". Tales resultados concuerdan con la investigación de Galeano et. al (6) en lo que corresponde a valorar el cuidado como "bueno" en un 57\%. Delgadillo (7) sostiene que el $61 \%$ de los individuos tiene "satisfacción media". López (8), en contraste, señala que el $32 \%$ de individuos se halla satisfecho en la dimensión técnica. Pueden evidenciarse tendencias variables en cuanto a la dimensión técnico-científica, lo cual puede deberse a las características en cuanto a los procedimientos.

Según la dimensión humana, la mayoría de padres percibe una buena atención por el personal de enfermería durante el cuidado de los niños; considerando que dichos pacientes requieren muestras de preocupación que de forma directa influyen sobre su estado físico y emocional. Lo señalado está representado por el $94,5 \%$ de individuos quienes consideran que "siempre" se desarrollan buenas prácticas en el trato. De acuerdo a esta dimensión, Guinea (9) refiriéndose a un aspecto interpersonal señala que el $47 \%$ de individuos considera el comportamiento del personal medianamente favorable y el $30 \%$ lo considera favorable. Moreno (10), también considerando el factor interpersonal, señala que el $53 \%$ de los individuos presenta un nivel de satisfacción medio y el $40 \%$ presenta un nivel de satisfacción alto. Ramos et.al (11) considera el factor "amabilidad" por el personal de enfermería, señalando que el $42,4 \%$ de individuos opina favorablemente sobre esta característica.

Según la dimensión entorno, la mayoría de los padres consideran que el ambiente en donde se llevan a cabo las actividades de cuidado por el personal de enfermería, es adecuado. Las características respecto a la opinión favorable acerca del ambiente de atención, se muestran entonces, como un factor predominante manifestado por los individuos cuyos hijos se hallan hospitalizados. Evidenciando de esta forma, la idoneidad de las instituciones, por cuanto controlan la presencia de contaminantes en el área específica. Delgadillo (7), señala que el $70 \%$ de individuos tiene satisfacción media respecto al entorno ya que apreciaban las instalaciones adecuadamente distribuidas, modernas y limpias. López (8) refiere que en la dimensión entorno, el $51 \%$ de individuos estaban medianamente satisfechos y el $22 \%$ se hallaban satisfechos. En la mayoría de caso, las 
condiciones del espacio en donde se desarrollan las actividades de atención por el personal de enfermería parecen ser apropiados, argumento cuyo sustento se evidencia en los altos porcentajes de opinión favorable.

Considerando el objetivo general del presente estudio: Determinar la percepción de los padres respecto a la calidad durante el cuidado de enfermería a pacientes pediátricos en el Hospital San Juan de Lurigancho, 2017, se evidencia que en la mayoría de los casos, los padres de familia perciben una atención apropiada por parte del personal de salud durante el cuidado de sus hijos hospitalizados, tal percepción se presenta de manera semejante con cada una de las dimensiones; es decir, el aspecto técnico científico, aspecto humano y entorno. En menor proporción, se evidencia una percepción no favorable, de que a pesar del valor reducido, es también importante por cuanto la aceptación a un tratamiento y posteriormente la recuperación de los pacientes obedecen a cada uno de los elementos en el ámbito específico y cómo estos son captados por los usuarios del servicio y su entorno personal cercano. Conforme a lo señalado, los resultados evidencian la tendencia de percepción favorable hacia la atención por el personal de enfermería. Galeano et.al (6) se refiere a tal tendencia, señalando que, el grado de satisfacción de los padres respecto a la atención de enfermería es satisfactorio influyendo positivamente en la utilización de los servicios y en el grado de conformidad con los cuidados. Guinea (9) concluye que el mayor porcentaje de los padres expresan una percepción medianamente favorable. En la investigación desarrollada por Marky (12) las conclusiones no son positivas ya que, el mayor porcentaje de los padres manifestaron un nivel de satisfacción medio o bajo y el cuidado por el personal de enfermería fue calificado bueno y en otros casos regulares. A pesar de las evidencias sobre la opinión favorable por parte de los padres de niños hospitalizados, y la preocupación por las instituciones hacia el control de los componentes de cuidado de los pacientes; es indispensable, identificar los casos desfavorables y evaluar las características involucradas directa e indirectamente en su manifestación.

\section{CONCLUSIONES}

La percepción de los padres respecto a la dimensión técnico-científica es favorable en la mayoría de casos. Asimismo, 1,a percepción de los padres respecto a la dimensión humana es favorable en la mayoría de casos. Con respecto a la percepción de los padres respecto a la dimensión entorno es favorable en la mayoría de casos. Finalmente, la percepción de los padres respecto a la calidad durante el cuidado de enfermería a pacientes pediátricos en el Hospital San Juan de Lurigancho es en la mayoría de casos, favorable.

\section{AUTOR DE CORRESPONDENCIA}

Victor Humberto Chero Pacheco

Universidad María Auxiliadora

Av. Canto Bello $N^{\circ} 431$

Lima 36 - Perú

Teléfono: $+51-1-3891212$

E-mail:vchp_dent@hotmail.com

\section{REFERENCIAS BIBLIOGRÁFICAS}

1. OPS-OMS. Estándares e indicadores para la acreditación de hospitales. [Online]. 1992 [citado el 3 de marzo 2017]. Disponible en: http://apps.who.int/medicinedocs/document s/s16603s/s16603s.pdf

2. García, María. Gestión de la atención al cliente/consumidor (UF0036) [Internet]. Málaga: IC Editorial; 2011. [Citado 14 enero 2017]. Disponible en: ProQuest Ebook Central

3. Cottle, D. El servicio centrado en el cliente: cómo lograr que regresen y sigan utilizando sus servicios [Internet]. Madrid: Ediciones Díaz de Santos; 1991. [citado el 14 de febrero 2017]. Disponible en: ProQuest Ebook Central

4. Lemus, J, Aragües y Oroz, V. Auditoría médica y profesional integral: la calidad de la atención en los servicios de salud [Internet]. Buenos Aires: Corpus Editorial; 2006. [citado el 14 de febrero 2017]. Disponible en: ProQuest Ebook Central

5. García, María. Gestión de la atención al cliente/consumidor (UF0036) [Internet]. Málaga: IC Editorial; 2011. [Citado 14 enero 2017]. Disponible en: ProQuest Ebook Central

6. Galeano H, Furlán C, Auchetr M, Balbuena M, Zacarías G y Zacarías L. Nivel de satisfacción con la atención de enfermería en un hospital pediátrico. [Internet]. 2006 [citado el 3 de marzo 2017]. Disponible en: http://www.unne.edu.ar/unnevieja/Web/cyt/ cyt2006/03-Medicas/2006-M-134.pdf

7. Delgadillo D. Satisfacción de la madre del recién nacido hospitalizado sobre la calidad del cuidado del profesional de enfermería en el Servicio de Neonatología del Instituto Nacional Materno Perinatal-2013. Ateneo 
UNMSM. [Online]; 2014 [citado el 10 de febrero 2017]. Disponible en: http://ateneo.unmsm.edu.pe/ateneo/bitstrea m/123456789/4309/1/Delgadillo_Morales_ Doris 2014.pdf

8. López B. Nivel de satisfacción de las madres de niños/as menores de 2 años sobre la calidad de atención del profesional de enfermería en el programa de crecimiento y desarrollo del Hospital Santa Rosa Lima Perú, 2014.Ateneo UNMSM. [Online].; 2014 [citado el 10 de febrero 2017]. Disponible en: http://ateneo.unmsm.edu.pe/ateneo/bitstrea m/123456789/4809/1/Lopez_Tirado_Bertha _Milagros_2015.pdf

9. Guinea, $\bar{M}$. Percepción de los padres de niños (as) menores de 5 años sobre la atención humanizada de la enfermera en el servicio de CRED en el Centro de Salud Mirones - 2016 cybertesis UNMSM. [Online]; 2016 [citado el 8 de enero 2017]. Disponible en: http://cybertesis.unmsm.edu.pe/bitstream/cy bertesis/5371/1/Guinea_lm.pdf

10. Moreno E. Nivel de satisfacción de la calidad de atención de enfermería de los padres de familia o acompañante del paciente pediátrico oncológico en el Servicio de Emergencia del Instituto Nacional de Enfermedades Neoplásicas: febrero-julio, 2009.Ateneo UNMSM. [Online]; 2013 [citado el 25 de enero 2017]. disponible en: http://ateneo.unmsm.edu.pe/ateneo/bitstrea m/123456789/3993/1/Moreno_Cabello_Viv iana_Elida_2010.pdf

11. Ramos V, Rico R y Martínez P. Percepción del familiar acompañante con respecto al cuidado de enfermería en hospitalización. Enfermería global [Internet]. 2012 Ene [citado 3 de marzo 2017]; 11(25): 219-232. Disponible en: http://scielo.isciii.es/scielo.php?script=sci_a rttext\&pid=S169561412012000100013\&lng=es

12. Marky G. Satisfacción según percepción de los padres y cuidado enfermero al niño con infección respiratoria aguda en el Hospital Nacional Dos de Mayo diciembre 2012. Cybertesis UNMSM. [Online]; 2013 [citado el 10 de febrero 2017]. Disponible desde: http://webcache.googleusercontent.com/sear ch?q=cache:http://cybertesis.urp.edu.pe/bits tream/urp/278/1/Marky_ga.pdf 